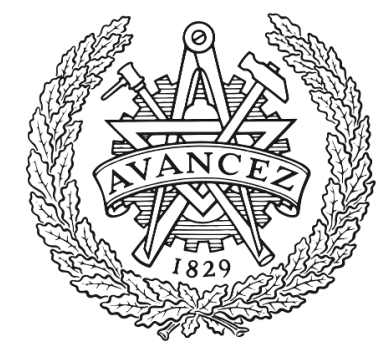

CHALMERS

UNIVERSITY OF TECHNOLOGY

\title{
A Ka-Band Active Integrated Antenna for 5G Applications: Initial Design Flow
}

Downloaded from: https://research.chalmers.se, 2023-04-26 15:29 UTC

Citation for the original published paper (version of record):

Liao, W., Maaskant, R., Emanuelsson, T. et al (2018). A Ka-Band Active Integrated Antenna for 5G Applications: Initial Design Flow. 2018 2nd URSI Atlantic Radio Science Meeting (AT-RASC). http://dx.doi.org/10.23919/URSI-AT-RASC.2018.8471330

N.B. When citing this work, cite the original published paper. 


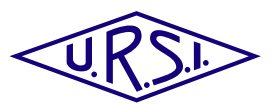

\title{
A Ka-Band Active Integrated Antenna for 5G Applications: Initial Design Flow
}

\author{
Wan-Chun Liao $^{(1)}$, Rob Maaskant ${ }^{(1)}$, Thomas Emanuelsson ${ }^{(1)(2)}$, Martin Johansson $^{(3)}$, Anders Höök ${ }^{(4)}$, Johan Wettergren ${ }^{(5)}$, \\ Michael Dieudonne ${ }^{(6)}$, and Marianna Ivashina ${ }^{(1)}$ \\ (1) Electrical Engineering Department, Chalmers University of Technology, Gothenburg, Sweden, http://chalmers.se
}

(2) Gapwaves AB, Gothenburg, Sweden

(3) Ericsson Research, Gothenburg, Sweden

(4) SAAB, Gothenburg, Sweden

(5) RUAG Space AB, Gothenburg, Sweden

(6) Keysight Laboratories, Keysight Technologies, Belgium

\begin{abstract}
Initial technology and design decisions are presented as part of a design flow to realize an active integrated antenna (array) element for 5G applications at Ka-band. The objective is to synthesize an antenna impedance that directly matches the amplifier optimal load impedance in order to maximize the overall efficiency of the integrated antenna-amplifier system while attaining an output power in the range of $15-25 \mathrm{dBm}$. A strategy to subsequently co-optimize this combined system is also discussed. Direct matching overcomes the insertion losses and bandwidth limitations of impedance-transforming networks, obviates the use of a standard 50-Ohm reference impedance, and potentially results in more cost-effective, more compact, and better performing active antenna designs in general.
\end{abstract}

\section{Introduction}

The continued growth in data traffic demands [1], both in terrestrial wireless and satellite communication applications, renders the present-day communication frequency bands inadequate in supporting these future demands. Solutions to handle upcoming capacity bottlenecks in mobile broadband services are thus needed, and the industry's focus is on utilizing frequencies above the conventional cellular sub- $6 \mathrm{GHz}$ bands, the mm-wave range in particular. At these higher frequencies, the available bandwidths can be on the order of $\mathrm{GHz}$, which offers the potential for both high system capacity and high peak rates.

A general link budget analysis of a wireless communication system reveals that the free-space path loss between transmitter and receiver exhibits quadratic growth as a function of frequency. The path loss is further increased by the higher atmospheric propagation loss at higher frequencies. This leads to a reduction in the link and system performance, which is further exacerbated by increased material losses and performance degrading factors of active semiconductor devices at higher frequencies. Obvious hardware/software solutions to improve the link budget are to (i) shorten the communication distance; (ii) increase the antenna gain; (iii) increase the absolute radiated output power, and; (iv) improve receiver sensitivity by decreased noise figure (NF) and increased receiver coding gain. Since a reduction in distance between transmitter and receiver is often undesired, impractical, or even impossible (in e.g. satellite communication applications), an increase in both antenna gain and absolute output power (i.e. effective isotropic radiated power, EIRP) is the solution of choice to partly mitigate the challenges at mm-wave frequencies. Large antenna gain requires a large radiating aperture. Hence, array antenna technology with integrated front-end electronics is therefore considered of key interest, also because it offers the possibility to perform electronic beamforming over a wide scan range.

Compact building practices are needed for mm-wave integrated array systems with many transceivers and antenna elements [1]. If modularity is required, the integrated front-end electronics must be confined within the cross-section of the aperture of the antenna element or sub-array of elements. Considering the significantly smaller area/volume of mm-wave products (dimensions scale with the wavelength), the complex interrelation between linearity, power efficiency, output power as well as minimum footprint size in the light of heat dissipation effects, clearly points to a need for novel multidisciplinary design methodologies that account for the interactions between different active and passive sub-systems.

Today, there is little to no well-defined multidisciplinary design flow focusing on the co-optimization of highly integrated antenna systems at mm-wave frequencies. This would involve a non-linear electronic circuit as well as an electromagnetic wave modeling approach, where the interface between the two respective sub-systems is aimed to be optimized from both circuit and antenna point-of-view. This requires full design flexibility both at the amplifier and the antenna level. The herein presented initial design flow is the first effort toward this goal, 
where the emphasis is made on the first optimization iteration of a single antenna (array) element co-designed with a pre-selected commercially available transistor to be configured as a power amplifier.

\section{System Performance Specifications}

Three application domains are considered that cover: (i) Space-based communication, in particular high capacity telecommunication systems at Ka-band, featuring a 30-GHz uplink and a 20-GHz downlink; (ii) Public safety and security, employing small array antenna systems for either fixed installations or unmanned mobile platforms (UAVs, road vehicles, and boats) in the 12 to $40 \mathrm{GHz}$ range, and; (iii) Terrestrial cellular wireless communication, employing e.g. 5G active mm-wave multi-user MIMO beamforming arrays. By harmonizing the inputs from these three application domains, a common aligned list of performance figures was created as shown in Table 1, which will hereafter be utilized as a baseline for our design flow in Sec. 3.

Table 1. Aligned system constraints of three application domains.

\begin{tabular}{cc}
\hline Parameter & Constraints \\
\hline Array aperture area & $10-100 \lambda^{2}$ \\
Array configuration & Planar array; $\sim \lambda / 2$ rect. grid \\
Radiating element & Dual-polarized \\
Frequency band & $20-40 \mathrm{GHz}$ \\
Bandwidth & $5-10 \%$ \\
Instantaneous bandwidth & $1-5 \%$ \\
Beamforming & Digital \\
Scan angle & $\pm \pm 60^{\circ}(\mathrm{EL} \times \mathrm{AZ})$ \\
Output power per element & $15-25 \mathrm{dBm}$ \\
Isolation between PAs & $-15 \mathrm{~dB}$ \\
Noise figure & $5-7 \mathrm{~dB}$ \\
Total power efficiency & $>25 \%$ \\
\hline
\end{tabular}

\section{Proposed Design Flow}

As explained above, a multidisciplinary co-design strategy is needed that can avoid (or minimize the use of) impedance-transforming networks, thus minimizing their insertion losses and overcoming bandwidth limitations by matching to optimal impedances, rather than to $50-\Omega$, while taking care of the heat dissipation problems at the same time. Fig. 1 describes the iterative optimization process of the preliminarily proposed design flow (electrical co-design only).

Regarding the power amplifier (PA), our choice of the integration level at this stage is to limit ourselves to a transistor-on-chip and to design the remaining circuitry off-chip as part of the radiating element. In future, the integrated circuit (IC) design of the transistors will be conducted in-house and the distribution of on- and

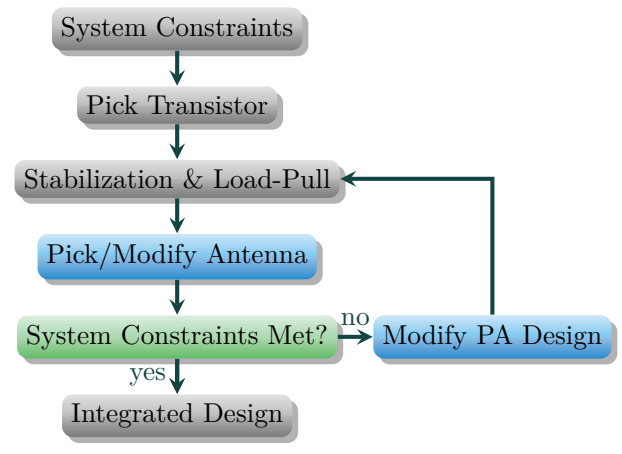

Figure 1. preliminarily proposed co-optimization flow chart.

off-chip components of the PA(s) can be chosen optimally. Furthermore, this paper describes only the first iteration of the flowchart in Fig. 1, i.e. from top to bottom, thus a suitable antenna is picked but not yet modified, which will be performed in subsequent iterations.

\subsection{Choice of Transistor}

The maximum available output power of semiconductor devices is intrinsically limited by the breakdown voltage of semiconductor technologies [1]; III-V compounds such as Gallium Arsenide (GaAs) and fast-growing Gallium Nitride $(\mathrm{GaN})$ materials are common design choices for mm-wave ICs owing to its relatively high peak output power [1,2], notwithstanding III-V materials are more expensive than silicon-based technologies and offer limited flexibility in terms of complex integrated designs. For these reasons, and together with the target specifications listed in Sec. 2, the GaN-based IC technology looks promising when it comes to a co-design of an active integrated antenna with a PA. For instance, a C-band GaN amplifier integrated active-antenna design with a maximum output power of $38 \mathrm{dBm}$ and a maximum PAE of $63 \%$ was demonstrated in [3]. Among a handful of commercially available off-the-shelf transistors, the Qorvo TGF2942 GaN on Silicon Carbide ( $\mathrm{SiC}$ ) high electron mobility transistor (HEMT) has an average maximum available gain greater than $10 \mathrm{~dB}$ between $20-30 \mathrm{GHz}$ for a drain-source voltage ranging from $V_{\mathrm{DS}}=20 \mathrm{~V}$ to $28 \mathrm{~V}$, and a $1-\mathrm{dB}$ compression point $\left(\mathrm{P}_{1 \mathrm{~dB}}\right)$ of $25 \mathrm{dBm}$ at $30 \mathrm{GHz}$, thus providing appealing characteristics for our applications.

A Modelithics HEMT model was used for the Advanced Design System (ADS) simulations. The DC-bias was determined by the static I-V characteristics of the transistor. A Class-A PA was designed during the first iteration in order to achieve high linearity first, implying that the antenna can have relaxed output filtering or harmonic tuning functionalities. The first iteration therefore reduces effectively to the trade-off between the amplifier PAE and the antenna radiation efficiency. The load-pull result for maximum $\mathrm{PAE}$ at $30 \mathrm{GHz}$ is presented in Fig. 2. The optimal load impedance has a relatively low 
resistance $(5.5 \Omega)$ and series inductive reactance $(29.2 \Omega)$ for a $49.7 \%$ PAE compared to $(17+\mathrm{j} 43) \Omega$ at the lower $18 \mathrm{GHz}$ frequency for a $56 \%$ PAE. The optimal load impedance approaches the edge of the Smith chart as the frequency increases towards $30 \mathrm{GHz}$, thus complicating the antenna-amplifier direct-matching due to the low optimal load resistance of the transistor.

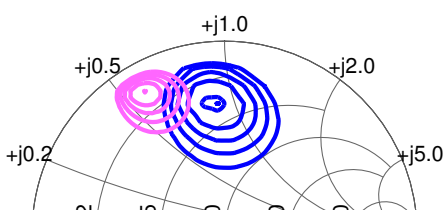

Figure 2. ADS simulated load-pull for maximum PAE for the HEMT at $30 \mathrm{GHz}$ (magenta, left) and at $18 \mathrm{GHz}$ (blue, right), contour interval: $5 \%$. (Test conditions: $V_{\mathrm{DS}}=$ $28 \mathrm{~V}, I_{\mathrm{DS}}=40 \mathrm{~mA}$, bondwires excluded.)

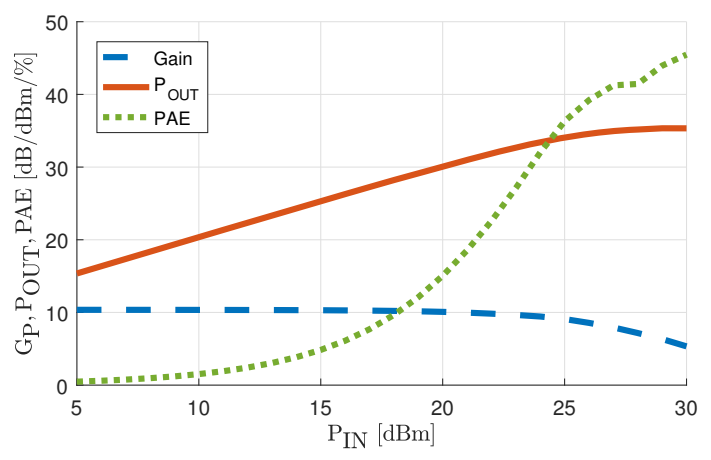

Figure 3. ADS simulated PAE, gain, and output power versus input power of the HEMT at $30 \mathrm{GHz}$. (Test conditions: $V_{\mathrm{DS}}=28 \mathrm{~V}, V_{\mathrm{GS}}=-1.3 \mathrm{~V}$, bondwires excluded.)

A K-factor stability analysis revealed that the PA needed an RLC stability circuit at the gate of the transistor (not shown) to reach an unconditionally stable condition and a conjugate match at the RF input source was implemented to enhance the transducer gain of the PA. Fig. 3 presents the ADS-simulated transducer gain, output power and PAE as a function of input power for the Qorvo TGF2942 GaN HEMT at $30 \mathrm{GHz}$, where the PAE is $15 \%$ with a gain of $10 \mathrm{~dB}$ at $5 \mathrm{~dB}$ back-off from $\mathrm{P}_{1 \mathrm{~dB}}$.

\subsection{Choice of Antenna Type}

We conclude from the load-pull results for maximum PAE at $30 \mathrm{GHz}$ ( $c f$. Sec. 3.1) that a low optimal load resistance of the PA indicates the PA output must be matched to an antenna with low radiation resistance and thus an electrically small antenna. Furthermore, an inductive optimal reactance of the antenna is desired to effectively compensate for the capacitive reactance at the output of the PA. Therefore, an active integrated antenna (AiA) comprising an electrically small loop type of antenna and a single-stage PA is considered without the need of using an impedance-transforming network. Small loop antennas

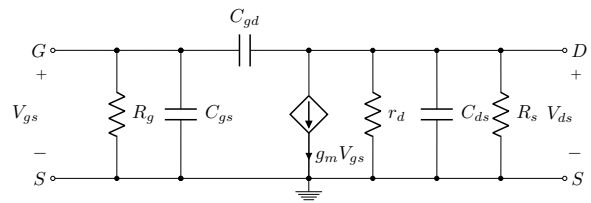

(a)

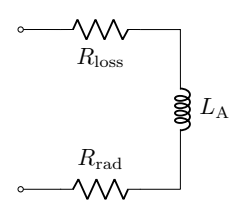

(b)
Figure 4. Equivalent circuit of (a) a common source amplifier, and (b) a small loop antenna.

can be economical and suitable for mass production when manufactured in printed circuit board (PCB) technology; however, PCB antennas suffer from low radiation efficiency when the radiation resistance becomes small with respect to its loss resistance.

A typical equivalent circuit of a common source amplifier is shown in Fig. 4(a) [4, p. 328], and an equivalent circuit model of an electrically small loop antenna is presented in Fig. 4(b). For a small loop antenna the antenna input impedance can be represented by $Z_{\text {in }}=\left(R_{\text {rad }}+R_{\text {loss }}\right)+$ $j \omega L_{\mathrm{A}}$, where $R_{\text {rad }}$ is the radiation resistance of the antenna, $R_{\text {loss }}$ represents the conduction-dielectric losses of the antenna materials, and $L_{\mathrm{A}}$ is the loop inductance. The radiation resistance of a single-turn circular loop antenna in free space can be written as [5, p. 209]

$$
R_{\mathrm{rad}}=\frac{\pi \eta k^{4} b^{4}}{6}
$$

where $\eta=\sqrt{\mu_{0} / \varepsilon_{0}} \approx 377 \Omega, k=2 \pi / \lambda$, and $b$ is the radius of the loop. The loss resistance $R_{\text {loss }}$ appears in series with the radiation resistance. Upon assuming that the wire radius $a$ is much larger than the skin depth and a uniform current distribution on the antenna, $R_{\text {loss }}$ can be represented as $[5$, p. 78]

$$
R_{\mathrm{loss}}=\frac{b}{a} \sqrt{\frac{\pi f \mu}{\sigma}}
$$

where $f$ is the frequency, $\sigma$ is the conductivity, and $\mu$ is the permeability of the metallic antenna material. The loop inductance can be written as $[5$, p. 216]

$$
L_{\mathrm{A}} \simeq \mu_{0} b\left(\ln \frac{8 b}{a}-2\right) \text {. }
$$

Finally, the radiation efficiency of the antenna can be written as $[5$, p. 78$]$

$$
\eta_{\mathrm{rad}}=\frac{R_{\mathrm{rad}}}{R_{\mathrm{rad}}+R_{\mathrm{loss}}}
$$

As an example, let us assume $2 \pi b=3 \mathrm{~mm}$, $a=0.0033 \cdot 2 \pi b$, and $\sigma=3.5 \times 10^{7} \mathrm{~S} / \mathrm{m}$, the analytic results for $R_{\text {loss }}$ and $\eta_{\text {rad }}$ as a function of $k b$ are shown in Fig. 5(a), where $\eta_{\text {rad }} \approx 26 \%$ as $k b=0.25$, which declines as the electrical size of the antenna decreases. Fig. 5(b) presents the analytic loop impedance and the numerical result simulated in a commercial EM simulator. The 


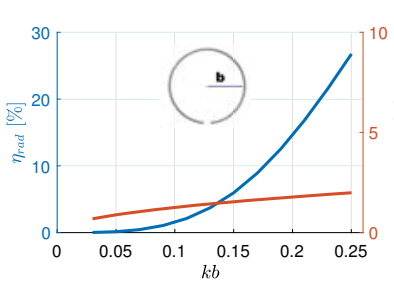

(a)

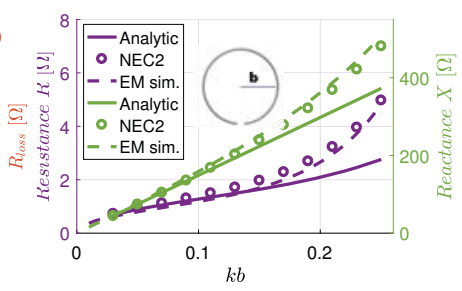

(b)
Figure 5. Radiation efficiency and impedance of small loop antenna based on analytic results.

simulated result is found around $(5+\mathrm{j} 500) \Omega$ and the analytic result is around $(3+\mathrm{j} 380) \Omega$ for $k b=0.25$; both the simulated and the analytic results have low resistances and suitable inductive reactances from the PA optimal load impedance point-of-view ( $c f$. Sec. 3.1). The discrepancies between the simulated and analytical results are caused by the herein employed analytic model, which is only valid as $k b<0.2$.

\section{Co-simulation Results}

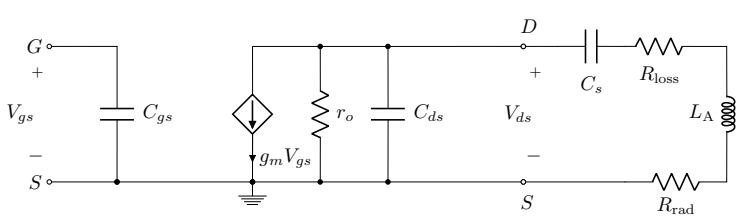

Figure 6. Small-signal equivalent circuit for the AiA.

Fig. 6 illustrates a small-signal equivalent circuit for the $\mathrm{AiA}$. The added series capacitor $C_{\mathrm{s}}$ at the output is a DC blocker needed to DC-bias the transistor at its drain. This capacitor also reduces the loop inductance seen from the drain pad to get it closer to the optimal PA load inductance without having to employ additional impedance matching circuits. For the above example, $\eta_{\text {rad }} \approx 26 \%$ for $k b=0.25$, and the PAE of the amplifier is around $15 \%$ at $5 \mathrm{~dB}$ back-off from $\mathrm{P}_{1 \mathrm{~dB}}$. Needless to say, this is only for a single design iteration and various improvements can be made on both the $\mathrm{PA}$ and the antenna designs in the subsequent iterations to enhance the efficiencies. Nonetheless, already now one can observe a trade-off between the antenna radiation efficiency and the optimal load resistance of the PA - a large antenna has higher $\eta_{\text {rad }}$, but also a larger radiation resistance, which in turn leads to a lower PAE.

\section{Conclusions}

A preliminary (co-)design flow for active integrated antenna applications has been presented. Three emerging applications in the $20-40 \mathrm{GHz}$ frequency range have led to the definition of an aligned set of performance specifications for which the GaN-based IC technology has been found most promising when it comes to a co-design of an active integrated antenna with a power amplifier
(PA) owing to its higher breakdown voltage leading to a higher output load impedance and thereby more optimal matching to the antenna impedance. Specifically, the Qorvo TGF2942 GaN HEMT was chosen in this work for its attributes to provide high output power and adequate efficiency when designed as a PA in the Ka-band. At $30 \mathrm{GHz}$ the PAE of the HEMT is $15 \%$ with a gain of $10 \mathrm{~dB}$ at $5 \mathrm{~dB}$ back-off from $\mathrm{P}_{1 \mathrm{~dB}}$. Furthermore, an electrically small loop antenna was considered to directly match its impedance to the optimal load impedance of the PA without using an impedance-transforming network. A single iteration in the design flowchart already revealed an interesting trade-off between the antenna radiation efficiency and radiation resistance (expressed analytically) on the one hand, which are affected by antenna dimensions, and on the other hand the optimal PA load resistance. Take the example in Sec. 3, $\eta_{\text {rad }} \approx 26 \%$ for the electrically small loop antenna $(k b=0.25)$ while the PAE is $15 \%$ for the PA. The $\eta_{\text {rad }}$ can be improved by increasing the antenna dimensions that would also lead to a deterioration of the PAE to be lower than $15 \%$, and vice versa. Further iterations are required to realize a co-optimized AiA design, such as adjusting the DC-bias for the PA to improve the PAE and modifying the antenna design to increase the radiation efficiency without compromising the PAE. The details of the co-optimized integrated antenna-amplifier design which has been realized using the proposed design flow will be shown at the conference.

\section{Acknowledgment}

This research has been carried out in ChaseOn Centre in Integrated Antenna Array project financed by Vinnova, Chalmers University of Technology, Royal Institute of Technology (Stockholm), Ericsson, Saab, Ruag Space, Keysight, and Gapwaves. Simulation models utilized under the University License Program from Modelithics, Inc., Tampa, FL and Qorvo, Portland, Oregon.

\section{References}

[1] Ericsson, "On mm-wave technologies for NR," Tech. Rep. R4-164226, 2016. [Online]. Available: http://www.3gpp.org/ftp/TSG_RAN/WG4_Radio/ TSGR4_79/Docs/R4-164226.zip

[2] — - "Ericsson Technology Review: Microwave Backhaul Beyound 100GHz,” Tech. Rep., 2017.

[3] N. Hasegawa and N. Shinohara, "C-Band Active-Antenna Design for Effective Integration With a GaN Amplifier," IEEE Trans. Microw. Theory Tech., pp. 1-8, 2017. [Online]. Available: http://ieeexplore.ieee.org/document/7976322/

[4] Adel S. Sedra and Kenneth C. Smith, Microelectronic Circuits. Oxford University Press, 2004.

[5] C. A. Balanis, Antenna Theory: Analysis and Design. John Wiley \& Sons, 1997. 